\title{
Psychosocial experiences of the internet in a group of adolescents: A qualitative content analysis
}

\author{
Mehrsadat Mahdizadeh ${ }^{1}$, Mahnaz Solhi ${ }^{2}$, Farbod Ebadifard Azar ${ }^{1}$, Ali Taghipour $^{3}$, \\ Aliasghar Asgharnejad Farid ${ }^{4}$
}

Received: 23 November 2016

Published: 20 Aug 2017

\begin{abstract}
Background: Social networking has a dramatically increasing trend among adolescents. By creating novel models of content production, distribution, and reception, this space has introduced opportunities and threats for adolescents, which must be understood in relation with their health status. This study was conducted with the aim of describing the psychosocial experiences of Iranian adolescents in the Internet's virtual space.

Methods: The present qualitative formal content analysis was conducted in Mashhad a city Iran. The participants included 32 adolescents of 13-18 years of age. Data were collected through 32 semi-structured individual and group interviews with maximum variation. The data were recorded, transcribed, and then analyzed via MAXQ 10 software.

Results: In this study, 2 main themes of "moving towards constructiveness" and "perceiving social and psychological tensions" were formed. Accordingly, 9 subcategories were formulated including: increasing the social capital, a good feeling in life, escaping loneliness, being seen in the social network, intelligent selection of content, perceived threats, temptation, decline of behavioral values and principles, and emotional and social helplessness.

Conclusion: Adolescents' positive and negative experiences in the Internet form based on personal and environmental factors. These experiences affect the mental and social dimensions of their health. These factors call for the attention of scholars and policymakers for developing enabling strategies for adolescents, and their families and for experts for promoting adolescents' health.
\end{abstract}

Keywords: Virtual space, Internet, Social networks, Adolescent, Health, Qualitative study, Content analysis

Copyright@ Iran University of Medical Sciences

Cite this article as:Mahdizadeh M, Solhi M, Ebadifard Azar F, Taghipour A, Asgharnejad Farid A. Psychosocial experiences of the internet in a group of adolescents: A qualitative content analysis. Med J Islam Repub Iran. 2017(20 Aug);31:46. https://doi.org/10.14196/mjiri.31.46

\section{Introduction}

Today, social networking in the Internet is an important part of social life (1). The use of social networks in the internet dramatically has increased among adolescents in the past decades. Adolescents are the most active users of social networks. Studies show that they spend a considerable part of their daily life interacting with social networks (2). Based on a survey in the Pew Research Center (2015), $89 \%$ of adolescents have reported the use of at least one and $71 \%$ reported more than one social network (3). Ac- cess to various virtual networks has become an epidemic among most Iranian social groups including the youth and adolescents (4), with numerous positive and negative outcomes for them. Social networks influence three aspects of adolescent's growth and development: the sense of belonging, psychological well-being, and the process of identity growth (5). With the emergence and popularity of the Internet, Internet-based networks known as social networks were introduced. Relations in the social net-
Corresponding author: Dr Mahnaz Solhi, solhi.m@iums.ac.ir

1. Department of Health Services and Health Education, School of Health, Iran University of Medical Sciences, Tehran, Iran

2. Health Promotion Research Center, Department of Health Services and Health Education, School of Health, Iran University of Medical Sciences, Tehran, Iran.

3. Health Sciences researches Center, Department of Biostatistics and Epidemiology, School of Health, Mashhad University of Medical Sciences. Mashhad, Iran.

4. School of Behavioral Sciences and Mental Health, Tehran Psychiatry Institute, Tehran, Iran. $\uparrow$ What is "already known" in this topic:

Findings provide an insight into how youth identity and experiences are formed in the Internet networked space. Also the advantages of online technologies have been reported for elevating self-confidence, perceived social support, social capital, experience of a certain identity, and opportunities for self-disclosure, and their harmful effects have been reported as increasing exposure to damage, social isolation, depression, and cyber-abuse.

\section{$\rightarrow$ What this article adds:}

Adolescents take part in virtual social networks according to their personal capabilities and social and family potentials. Virtual socializing experiences may have a significant effect on various dimensions of adolescents' health. 
works have created a novel, expansive, and variable environment which is self-productive in content, selfregulating in distribution, and self-selecting in reception by the audience (6).

Recent innovations in the Internet's virtual space, with their numerous and extensive functions, have introduced challenges as well as benefits and welfare for humans. The motivation for many such innovations is providing welfare and comfort and strengthening human values in order to achieve perfection; nevertheless, the misuse of these tools has occasionally harmed people's body, morality, and soul, and transformed their cultural values and social behaviors (7).

The Internet has an important impact on the sociocultural structure of contemporary societies and Iran has also been influenced by this factor. In today's world, new communication technologies have created enormous changes and complex in human relations and created a new form of behavior patterns (8). Cyberspace provides an opportunity to interact with individuals and social groups with the diversity of thoughts and beliefs, traditions, languages and religions in this space (9). The family plays an important role in Iranian culture (10). Due to the effects of virtual socializing, behaviors and attitudes such as cultural beliefs and norms have changed in the Iranian society, especially the younger generation (9).

In this space, people build friendships and enmities and share experiences of actual world (11). These networks create paradoxes for social relations. On the one hand, they facilitate online groups and relations, and on the other, they are a source of isolation and exclusion (5).

Zare et al. has demonstrated the perceptions of Iranian female and male university students of the destructive effects of cell phone-based virtual social networks on family, moral, cultural, religious, mental, and physical dimensions (4). Another study on the unwished experiences of the youth has shown sexual solicitation and online abuse as threats to the users (12). Findings of Davis in 2016 provide an insight into how youth identity and experiences are formed in the Internet's networked space. As a response to a preplanned scenario, participants spoke of the advantages of multiple online profiles, mentioned mental health issues and hateful discussions, and pointed to the fact that they could express themselves more freely in online spaces than in offline spaces such as the school (13). The youth receive different information from various sources including new communication technologies, especially the Internet, which results in the emergence of virtual networks and, consequently, social capital. The constructed social capital could strengthen the relations. However, the information resulting from these networks is modified and reproduced within communities. The youth and adolescents make use of available sources and select from among them in order to continually define and redefine their identities. The virtual space, especially the Internet, which is partly responsible for the process of globalization, provides for youth the opportunity for communicating without the supervision of others, especially parents, and can potentially affect the process of socialization and role relations among the youth and adolescents
(14).

Some researchers analyzed psychological aspects of the Internet from a negative viewpoint; however, it should be pointed out that the cultural concepts shared in this space create a mental environment which facilitates excellence and prosperity by providing opportunities for thought and experience (15). In a systematic study, the advantages of online technologies have been reported for elevating selfconfidence, perceived social support, social capital, experience of a certain identity, and opportunities for selfdisclosure, and their harmful effects have been reported as increasing exposure to damage, social isolation, depression, and cyber-abuse (16).

In the Internet knowledge, skills, and behavioral and moral rules are constantly questioned. In such conditions, adolescents who look for gaining new experiences and discovering themselves face some complexities in this space. Various factors influence the life and socialization of adolescents, and the Internet exerts a wide spectrum of such influences on adolescents. Although the Internet has an influential role on all aspects of adolescents' life, no deep qualitative study has yet been conducted on Iranian adolescents in this regard. Most qualitative studies have been conducted on the young people, and studies on adolescents have mostly been quantitative and focused on a single dimension of this new phenomenon including the psychological dimension. To understand different dimensions of this phenomenon, the present study aimed to describing the psychosocial experiences of Iranian adolescents in the Internet's virtual space.

\section{Methods}

Design

This research is a qualitative formal content analysis. Content analysis is the method of analyzing written, spoken, or visual messages, and the raw data resulting from these analyses are summarized and categorized. In formal content analysis, categories and their names emerge from the data (17).

\section{Setting and Participants}

The sampling began in March 2015 and ended in September 2016 in Mashhad, Iran. The participants of this study were selected using purposive sampling. Sampling was performed on female and male adolescents with maximum variation in level of education, social and economic status, and age, until the data were saturated with the participation of 32 adolescents. Inclusion criteria were being adolescent (13-18 years old) and Iranian. The interviews were conducted in the natural life environments such as schools, parks, cultural centers, and online game clubs, which facilitated access to adolescents.

\section{Data collection}

Data were collected through semi-structured individual (18 teenagers) and group face-to-face interviews (2 group discussions with 18 participants).Sampling was done by taking the maximum variance. The interviews began with the general topic, "Tell us about your experiences with using the Internet's virtual space" and continued with fol- 
low-up questions such as "What do you mean?" or "Can you explain more?" to collect more information. Each interview lasted for 50-110 minutes and was recorded with the participants' informed consent. Group interviews were conducted in two separate groups for girls or boys, with 8 people each, and lasted for 90-120 minutes.

\section{Data Analysis}

The interviews were first transcribed on paper and, after multiple thorough reviews, turned into semantic units. These semantic units were then summarized, resulting in codes and categories, and categories which repeated systematically led to themes. Data analysis was performed in parallel with data collection.

\section{Trustworthiness}

Long engagement, triangulation in data collection (recorded interviews, group discussions, and notes), reviewing observer revisions, and continuous comparison of data were used for data validation. Dependability demonstrates the reliability and consistency of data. For this purpose, we used member checks and external checks. Rich description of data contributed to the transferability of the study (18).

\section{Ethical Considerations}

Ethical considerations in the study included obtaining written informed consent forms from the participants, anonymous recording of the interviews, privacy of the information resulting from their experiences, and freedom to exit the study whenever they want.

\section{Results}

Thirty-two 13-18-year-old adolescents (16 girls and 16 boys) participated in this study. Twenty-eight $(87.5 \%)$ were students and $4(12.5 \%)$ were not. Five participants $(16 \%)$ lived with one of their parents because of divorce or the death of one parent. The dimensions of their families were 1-7. The themes emerged after data analysis, which were including 2 main themes of "moving towards constructiveness" with 5 sub-categories and "perceiving social and psychological stress" with 4 sub-categories (Table 1).

\section{Moving towards constructiveness}

A number of participants use the virtual world of the Internet in a constructive and forward-moving path. They said that using the Internet had positive outcomes in different dimensions of their lives, such as providing a good

Table1.Major themes and sub-categories emerging from adolescents' experiences

\begin{tabular}{ll}
\hline Major theme & Sub-categories \\
\hline Moving towards & Having a good feeling \\
constructiveness & Increasing the social capital \\
& Being seen in the social network \\
& Escaping loneliness \\
& Intelligent selection of content \\
Perceiving social & Temptation in the Internet's space \\
and psychological & Emotional and social helplessness \\
tensions & Perceived threats \\
& Decline of behavioral values and principles \\
\hline
\end{tabular}

feeling in life, increasing the social capital, being seen in the social network, escaping loneliness, and intelligent selection of content.

\subsection{Having a good feeling}

Most participants suggested that they encounter different things in the Internet's virtual space and gain much information, which help them have a good feeling in life. For example, contents, films, and advertisements which inform them of everything around the world give them a sense of being up-to-date and guide them towards constructiveness by increasing their self-confidence, providing opportunities for the development of their social roles, and giving them a good feeling. Participants stated:

"Virtual spaces help us live better and learn about everything. That's what life is all about. How can we live without these? Life is being online and up-to-date. I get a positive energy and a good feeling. I can do things such as designing my house, learning about clothes and haircut fashions, brands of cars, and lots of other things. Telegram is good for communication; I am in touch with my friends; it also has many other features which make me feel up-to-date. On the other hand, Facebook, Instagram, and Twitter bring me the world news and give me the feeling of being up-to-date, which is good for me" (Participant 11, 14-year-old boy).

"Well, I learn about new discoveries in the virtual world which is really good for life; good moral messages help me learn a lot and open my mind" (Participant 1, 18-yearold girl).

"I have Telegram. There are messages and pictures sent there or elsewhere online which could boost our morale, really increase our self-confidence, make us select a goal for ourselves, and think about our future, and these help us become a successful person for ourselves and the society" (Participant 4, 14-year-old girl).

"Internet and Telegram have helped me a lot with my social activities; they have helped me with publishing some news I have prepared" (Participant 5, 16-year-old girl).

\subsection{Increasing the social capital}

While speaking of their experiences in the Internet's virtual space, participants mentioned the social support they had gained through encouragement and sympathy, and also the facilitation of social relations which had had a positive effect on them.

"Well, I am a member of social networks. People message me and say, 'Bravo for your behavior'; they encourage me; so I will post more" (Participant 10, 17-year-old girl).

"I use virtual social networks to attract my classmates and expand my friendships and social relations" (Participant 8, 18-year-old boy).

"I think it's better to use social networks, since I have found long-lost friends which felt good" (Participant 22, 18-year-old boy).

\subsection{Being seen in the social network}

Self-assertion, satisfaction with playing a role, and be- 
ing influential in the network of social interactions are among the experiences of participants in the Internet's virtual world which give them a feeling of pleasantness, worth, and self-confidence.

"I have set a picture of hijab as my profile picture and post my own writings. Many say, 'You know and understand more than your age". They say, 'You can decide what to do', which is pleasant for me" (Participant 10, 17 year-old girl).

"When I reasonably argue with my friends and family in Internet networks, they count on me more and say, 'He understands a lot and we can count on him." (Participant 7, 18-year-old boy).

\subsection{Escaping loneliness}

Most participants complained of loneliness, mostly because their parents worked or did not pay attention to them, the interaction gap with their parents, small family dimensions, and lack of recreational facilities. To escape loneliness and achieve a good feeling in life, they took refuge in the virtual world of the Internet.

"One of the advantages of networks and Telegram is that they are entertaining. There are not enough recreational and sport facilities, or they are expensive. It is expensive to go to a gym or pool" (group interview, Participant 31, 17-year-old boy).

"I am always alone. I'm not allowed to go out. My mom works late. I use virtual networks in Telegram mostly for escaping loneliness and communicating with my friends" (Participant 14, 16-year-old girl).

\subsection{Intelligent selection of content}

Most participants make intelligent selection of content on the Web and follow contents which lead them towards constructiveness, with an understanding of positive and negative aspects of the Internet's virtual space as a double-edged sword, with skills of media literacy, and by relying on family and environmental capacities.

"Well, it's a double-edged sword; it depends on us how to use it; if we have definite goals for our lives, we'll use it according to those goals. I have a goal; I want to be helpful for my family and society" (Participant 17, 13-year-old girl).

"I used to think the Internet and virtual networks were bad; but now I see they are good, if we think positively and do not follow bad things. If we try to use them well, nothing bad will happen. I use good texts and pictures people send and try to behave and talk accordingly" (Participant 20, 14-year-old girl).

"I have principles in my mind and am committed to behave accordingly in these social networks" (Participant 5, 16-year-old girl).

"I try to behave properly in virtual networks where boys and other people are members. I try to not behave such that they would abuse me or behave as they wished. I behave such that I can keep my dignity" (Participant 10, 17year-old girl).

"Three years ago I met two or three girls on a network called Nice One. Even back then, I had boundaries based on the performance and values of my family. I didn't get numbers from anyone. I respect my own boundaries and try to have a calm and good life" (Participant 8, 18-yearold boy).

\section{Perceiving social and psychological tensions}

The experience of virtual world has brought about extremely stressful conditions for some participants. They had encountered temptations, perceived numerous threats, and experienced emotional and social helplessness. Moreover, many asserted that the behavioral principles and values that had been important to them have faded over Internet virtual interactions.

\subsection{Temptation of the Internet space}

The feeling of temptation of the Internet's virtual space was one of the sub-categories of perceiving mental and social stress, emerging from the experiences of participants. Most participants stated that inclination towards new experiences and their pleasures have tempted them to act upon them. Moreover, a feeling of catharsis and relief from mental and psychological conflicts was important for participants. They considered the Internet's virtual space as a haven for escaping sexual needs and easing their discomforts to gain tranquility, because they faced limitations in the release of emotions.

"I want to experience and enjoy. In the virtual space, I noticed addictive drugs are used and I tried to use as well, but I felt disgusted and didn't continue to use anymore. The next time I saw and experienced something else" (Participant 29, 17-year-old boy).

"Some Internet networks and contents are tempting, for example those which teach sex. I am tempted to open them. Temptation has happened to me a lot. As soon as I type a word on the Internet, I see the +18 sign. This is really tempting, and I want to open the link with a proxy to see what it is" (group discussion, Participant 17, 15year-old boy).

"We are facing a fashionable world in the virtual space which has changed our behaviors under the influence of people in virtual networks and made us take up behaviors which others call 'open-mindedness'. This is promiscuity" (group interview, Participant 27, 14-year-old girl).

"There are Telegram channels which have endangered all dimensions of our lives, because they are tempting and vastly advertised. We are thirsty for opening any picture we see" (group interview, Participant 23, 16-year-old boy).

"If I am sad with daily activities and being busy, I turn to them to calm down. Or when I feel lustful, I want to satisfy it, so I turn to them" (Participant 9, 13-year-old boy).

\subsection{Emotional and social helplessness}

The realities perceived by adolescents in the virtual world include emotional separation from their families, emotional gap in the Internet's virtual world, distancing from the real life, and decrease in social interactions, which result in oblivion to self and others and bring about a sense of emotional and social helplessness for the participants. 
"There is some kind of emotional divorce in my family. I am distancing from my mom, and my direct relations with friends and relatives has become limited" (group interview, Participant 25, 15-year-old girl).

"We leave the real world behind because the Internet's virtual world, like Clash, is addictive. They have decreased our actual relations. Virtual relations have distanced us from everything" (group interview, Participant 32, 17-year-old boy).

"Telegram is addictive. It makes we fall behind our life. It increases mental conflict. The goals seem unimportant to us and we do not follow them. We lose relations with the family. It has also reduced face-to-face social interactions" (Participant 5, 16-year-old girl).

"Relations in networks are devoid of feelings. Relations have also decreased. I see my neighbor, say hi, and pass. I do not interact with them anymore. These things affect my morale and I can't turn back. I easily make friends in these networks, but our relationships are useless and we break up after a couple of weeks. Social relations are better, with a higher affective load and happiness in the real world" (Participant 12, 16-year-old boy.)

\subsection{Decline of behavioral values and principles}

Adolescents stated in their experiences that they face contents in the Internet's virtual space that have brought about a decline in behavioral values and principles they had been committed to before. They say:

"Foreign role-models are extremely influential. We have many complexes because of the limitations, and we face paradoxes in the society. We see actresses wear hijab in movies, but when their pictures go viral in networks, we see they don't. We have forgotten our origins and lost our values because of people on the Internet and in virtual networks" (group interview, Participant 21, 16-year-old girl).

"It's like a trap. The further you go, the further you want to go. It feels good. I am like this now. I have learned many things I hadn't been familiar with. Many good behaviors I had based on my values and my family's efforts are gone and are not valuable to me anymore. Bad behaviors and foolery which I had never had are now quite normal to me" (Participant 16, 14-year-old boy).

\subsection{Perceived threats}

Encountering a huge amount of information and various interactions in the Internet's virtual space is one of the challenges adolescents face and among factors contributing to the sense of stress in participants, forcing them to face immoral matters and experience a sense of dependence, deficiency, routine, and decline.

"Misuse and participation in different channels and groups in Line has taken much of my time. My studies were really important, but I scarified them for this. Gradually, my first-semester grades became poorer. This has stopped me from progress" (Participant 1, 18-year-old girl).

"Well, 70\% of these social networks are important for me now. We can connect to everyone easily and see vulgar contents. I even had access to illegal or false contents in different channels of the virtual network and the Internet. These have certain effects. I feel I'm in a new condition at this age. It is a special age. These things can influence people of my age and in a bad way" (Participant 16, 14year-old boy).

"The use of Internet causes routines and repeated behaviors. It feels bad that some behaviors become routines. It makes you feel declining and stagnant" (group interview, Participant 26, 17-year-old girl).

\section{Discussion}

The Internet's virtual world is coupled with a wide spectrum of experiences for adolescents, such that they can either move towards constructiveness in this space, or face excessive tensions.

The Internet and its virtual social networks has provided new opportunities for gaining experience, having social interactions, encountering a world full of diverse contents, having a good feeling for life, escaping loneliness, increasing the social capital, being seen in the social network, and growing with intelligent selection of content for the adolescents participating in this study.

Providing an opportunity for the development and maintenance of relations in the online environment is one of the positive aspects of online technology, especially for the adolescents (5). Studies show that the use of social networks can be conducive to the psychological and social growth and well-being of adolescents $(5,19)$. Vossen et al. demonstrated that the use of social networks improved the capability of adolescents in understanding and sharing their emotions with their peers and enhancing their psychological health (20).

Social relations and connections are considered as a person's social capital and asset through which one can have access to the sources and supports available in these connections (14). The use of Internet facilitates for social relations (21). The development and facilitation of social interactions and gaining social capital were among reasons mentioned in the participants' experiences for using the Internet's virtual networks. As an interactional space, the Internet's virtual space reflects the same volume of social capital people possess in real life. The youth and adolescents are active in Internet's virtual networks in order to be connected with their real friends. These networks provide the opportunity for reviving old friendships despite physical distance. The members of Internet social networks experience a sense of belonging to the community and sharing interests and opinions, and enjoy it (22). The use of virtual social networks is directly related to gaining social capital by adolescents and predicts their citizen participation (23). In addition, Internet's virtual networks can remove the obstacles on the way of adolescents' selfconfidence by providing them with social capital (24). In a study by Li et al., (2015) conducted on students of a major state university in the US, Facebook interaction showed a positive correlation with social support. It was demonstrated that the different dimensions of social support could have various effects on the students' well-being (25). The number of friendships in virtual social networks positively influences supportive interactions, and per- 
ceived social supports are coupled with a sense of socialization and life-satisfaction (26). Membership and participation in the Internet's space increases the number of communicative networks, membership in different groups, complying with norms and rules, confidence in individuals and groups, gaining information and support, and access to various sources, which are all constitutive elements of social capital. The enhancement of social capital in Internet's virtual networks has an increasing effect on positive experiences and feelings, and a decreasing effect on negative ones, which eventually enhances happiness (27).

The adolescents' experience of being seen in social networks is in line with the results of other studies which have demonstrated that spending time in Internet's virtual networks results in viewing oneself more positively (28). Strengthening social contacts and the sense of emotional relations with others are the other positive outcomes of engagement with Internet network spaces. Most online interactions of the youth save their friendships and enhance their sense of intimacy (29). Wang et al., (2014) showed that the social function of this medium is one of the reasons for its use and brings about a sense of wellbeing in users (30). Presence in chat rooms, making friends, weblogs, virtual social networks, presenting attitudes and feelings in this environment, and the opportunity for free expression are among reasons why these networks are popular among the Iranian youth (31).

A study showed that receiving positive feedbacks on Internet profiles have an impact on psychosocial wellbeing including identity, self-esteem and relationship formation (32). Studies suggest that self-assertion to peers can enhance personal identity and intimacy among the youth. Self-assertion can help them solve issues they face, such as identity achievement, in order to move into adulthood. The youth have a unique opportunity to express their profession, religion, and political views in this space, which specifies identity achievement in the youth and adolescents (21). A longitudinal study demonstrated that the use of the Internet's virtual networks and online selfassertion have a bidirectional effect. The effect of selfassertion on the use of virtual social networks is socialization, and the effect of self-assertion on virtual social networks is selectiveness; these effects mediate the achievement of social capital for the users (30).

The feeling of loneliness was the chief complaint of adolescents participating in this study. They stated that they felt alone because their parents worked or did not pay attention to them, the interaction gap with them, small family dimensions, and lack of recreational facilities, and that they took refuge in the virtual world of the Internet to escape loneliness and achieve a good feeling for constructiveness. They enumerated being entertaining and helping them escape loneliness as the advantages of Internet's virtual space which caused them happiness. Trepte et al., (2013) showed that most virtual interactions of the youth are for entertainment (33). The youth consider activities in virtual networks as an appropriate way to help them escape loneliness and fill their spare time (22). Kearney et al., (2012) reported that adolescents present their Face- book interactions on the same friendly level of their faceto-face experiences (34) and feel less lonely (35). The results of a study indicated that loneliness and anxiety predict the individual's contact with others on Face book virtual network (36).

Adolescents participating in the present study asserted that they actively make use of media literacy skills, make intelligent selection of content, and use that space for moving towards constructiveness by setting goals for their lives, thinking positively while encountering negative aspects of the Internet's virtual space, trying to maintain their dignity in the virtual space through correct and valuable interactions, adherence to moral and behavioral values shaped in the family by their parents, and learning from past negative experiences in the virtual space. Through their media literacy skills, the audience increases their knowledge and potential for receiving information using the media and, thus, enhances critical thinking and turns the passive unidirectional relation with the media content into an active bidirectional interaction (37). Results of studies suggest that media literacy is a protective factor from smoking (38), drinking, and other high-risk behaviors in the adolescents. Teaching media literacy to adolescents can improve their critical analysis of media contents and their use, decrease violence, and affect all the dimensions of their health (39). A study showed that adolescents with a high degree of socialization in their offline and online life had the highest level of self-concept, and that online social life had managed to increase the level of self-concept, leading to social success (40). Another study suggested that collecting information from the Internet correlated with the sense of prosperity (41). The results of a cross-sectional study on Italian adolescents demonstrated that the Internet is one of the major factors which shape identity and personal independence and develop constructive relations outside the family in adolescents, allowing them to develop their potentials for identity discovery and providing a foundation for them to act independently (42).

A systematic review of the results of recent studies shows that the Internet technology can provide more opportunities for adolescents because of emotional relations with friends and school, while other studies suggest that the use of online technologies can also result in feelings of loneliness, depression, and social anxiety in the youth and pose mental health challenges for the adolescents (43). Individuals understand themselves in online interactions, try to view their effects on others by understanding their perceptions and, if needed, manage them by different mechanisms and change their perception of others by collecting data in the Internet's virtual space. Computer communications challenges not only impressionability management, but also the perception of self in others' eyes. Impressionability management over the Internet is similar to rowing a boat with two shovels in a stormy river which, without skill and social understanding, may endanger different dimensions of the adolescents' health (15).

"Perceiving mental and social tensions" was another major theme of this study with the sub-categories of temptation, perceived threats, emotional and social helplessness, and decline of behavioral values and principles. 
While sharing their experiences, the adolescents participating in the present study unveiled the tensions they faced in the virtual world. They struggled with a vast amount of tempting information and interactions and faced threats and difficulties in the dimensions of their health.

The Internet is a safe haven for many adolescents to escape problems. Online relations allow and individual to create new selves for people they may not meet again, which causes confusion; nevertheless, the adolescents are stimulated to engage in online relations (19). The youth incline towards attraction, being up-to-date, and the opposite sex, and any product, service, brand, or message which lets them feel revitalized attracts them because it strengthens their confidence and belief in themselves (44). Virtual networks facilitate the emotional experiences limited and forbidden by formal and common forms in the Iranian society, including friendship and interaction with the opposite sex, for the youth and adolescents (22). A number of adolescents participating in this study are tempted to use this space because of its special attractions with regard to friendship with the opposite sex, while understanding the cultural and social limitations of the society. Sexual need has long been a major topic in human studies and has affected humans' behaviors by occupying their minds throughout history. In line with other societies, it is evident that values, and thus sexual values, have changed in our society. Globalization generally and the effect of social networks specifically have transformed the perception of sexual needs' satisfaction, and individuals have - knowingly or unknowingly - formed new roles of sexual interactions in the Internet's space such as friendship between girls and boys, simultaneous multiple relationships, friendship with the opposite sex, and uncommon relations including sexual relations (45).

Dependence on the Internet is one of the concerns of adolescent mental health. In a study compatible with the experiences of adolescents in the present research, a positive correlation was observed between Internet use and addiction to it. The use of online games, Internet Apps, Twitter, and other online virtual networks increases the risk of Internet addiction (46). A qualitative study on Iranian young university students has demonstrated that social networks pose serious family, moral, cultural, mental, and physical threats to young users (15). Inclination towards the use of narcotics and alcohol is among the issues faced by young Internet users, in line with the experiences of adolescents in the present study (36). Online virtual networks promote the culture of using narcotics and alcohol, and the youth are tempting factors for this use by sharing pictures of their drinking and expression their sensations (47). Researchers have shown that low self-control in adolescents is in negative correlation with parents' positive support, aggravating Internet addiction in the adolescents (48).

The use of social network site led to cultural alienation in many societies (49). Similarly, in the present study, adolescents have confessed that Internet virtual interactions have resulted in the decline of their behavioral principles and family and social values.
Some participants have stated that overuse and misuse of the Internet may decrease family and social interactions and result in social seclusion. Yao et al., (2014) indicated that online social contact cannot be a good substitute for offline social interactions, and increasing face-to-face contact may help decrease the symptoms of Internet addiction (50). Low self-confidence (51), dissatisfaction with life (26), and being threatened and judged are correlated with extensive use of social networks, and these experiences affect the mental and social health of adolescents. Virtual social networks provide valuable support for the adolescents; on the other hand, they cause them negative experiences (52). The present study was the first to investigate the Iranian adolescents' experiences in the Internet's virtual space. One of the contributions of this study is that attention to the adolescents' experiences helps understand the positive and negative aspects of their interactions in the Internet's virtual space, which can aid scholars and policymakers in promoting health, and education experts in developing enabling strategies for the adolescents.

\section{Conclusion}

The use of Internet leads to social, psychological, and educational benefits as well as health issues for the adolescents. Adolescents take part in virtual social networks according to personal capabilities and social and family potentials and merits and experience various outcomes which may have a significant effect on different dimensions of their health.

\section{Acknowledgments}

The present article is a result of a research project (No. 94-04-27-27098). The authors would like to acknowledge the financial support of the Vice-chancellor for Research and Technology in Iran University of Medical Sciences, and all the adolescences who participated in the study.

\section{Conflict of Interests}

The authors declare that they have no competing interests.

\section{References}

1. Lai LS, To W. Content analysis of social media: A grounded theory approach. Electron Commer Res. 2015;16(2):138.

2. Ahn J. The effect of social network sites on adolescents' social and academic development: Current theories and controversies. J Am Soc Inf Sci Technol. 2011;62(8):1435-45.

3. Chandra A. Social networking sites and digital identity: The utility of provider-adolescent communication. Brown Univ Child Adolescent Behav Lett. 2016;32(3):1-7.

4. Zare RK, Salehi K. A Representation of Mobile Social Networks Damages for College Students: A Qualitative Study. Int J Humanit Cultur Stud. 2016;1(1):2562-74.

5. Allen KA, Ryan T, Gray DL, McInerney DM, Waters L. Social media use and social connectedness in adolescents: The positives and the potential pitfalls.As Edu Dev Psycho. 2014;31(1):18

6. Aghili SW, Mzadeh GIG. Social media; the nature, functions and challenges. J Iran Soc Dev Stud. 2016;8(1):21-36.

7. Rasouli M, submission SS. Content analysis of cultural and social content on social networks. J Cult Commun. 2013;15(59):67-85.

8. Shaeidi A. The role of information technology in cultural technology with a focus on: Developing the Islamic -Iranian culture. Media J Stud. 
2011;5(2):45-51.

9. Tajik MR, Kazemeini N. Relationship between cyberspace (internet) and cultural and religiusechenges in users.. J Cult Manag 2012;7(22):18-30

10. Ameli S, Hasani $H$. Two spaces damage and abnormalities cyberspace: comparetive study of international policy. J Cult Res. 2011;5(1):1-30.

11. Zndvanyan A, Heidari M, Bagheri R, Atarzadeh F. Cyberspace injuries among female students. J Cult Commun. 2012;14 (55).

12. Priebe G, Mitchell KJ, Finkelhor D. To tell or not to tell? Youth's responses to unwanted internet experiences.Cyber Psycho. 2013.

13. DavisK. Tensions of identity in a networked era: Young people's perspectives on the risks and rewards of online self-expression. New Media Soc. 2012;14(4):634-51.

14. Khajehnoori B, Delaware M. Factors affecting girlfriend and son among youth in Shirazwith an emphasis on globalization. Appl SociolJ. 2012;23(2(46)):41-64

15. Mortazpour M, Shahrbabaki ME, Tehrani HM. The relationship between the usage of social networking on life style and the level of self-esteem people in the city of Kerman. Int J Humanit Cult Stu. 2016;1(1):748-54

16. Best P, Manktelow R, Taylor B. Online communication, social media and adolescent wellbeing: A systematic narrative review. Child Youth Serv Rev. 2014;41:27-36.

17. Lewis S. Qualitative inquiry and research design: Choosing among five approaches. Health Promot Pract. 2015:1524839915580941.

18. Noble H, Smith J. Issues of validity and reliability in qualitative research. Evid Based Nurs. 2015;18(2):34-5.

19. Valkenburg PM, Peter J. Social consequences of the internet for adolescents a decade of research. Curr Dir Psychol Sci. 2009;18(1):15.

20. Vossen HG, Valkenburg PM. Do social media foster or curtail adolescents' empathy? A longitudinal study. Comput Human Behav. 2016;63:118-24

21. Pempek TA, Yermolayeva YA, Calvert SL. College students' social networking experiences on Facebook. J Appl Dev Psychol. 2009;30(3):227-38.

22. Pour-Samad A, Myrmohmdtbar SA, Afshar S, Sohrabi M. Community social capital network effects on girls and women in Tabriz. J Sociol Youth Stud. 2015;6(19):99-120.

23. Xie W. Social network site use, mobile personal talk and social capital among teenagers. Comput Human Behav. 2014;41:228-35.

24. Steinfield C, Ellison NB, Lampe C. Social capital, self-esteem, and use of online social network sites: A longitudinal analysis. J Appl Dev Psychol. 2008;29(6):434-45.

25. Li X, Chen W, Popiel P. What happens on Facebook stays on Facebook? The implications of Facebook interaction for perceived, receiving, and giving social support. Comput Human Behav. 2015;51:106-13

26. Oh HJ, Ozkaya E, LaRose R. How does online social networking enhance life satisfaction? The relationships among online supportive interaction, affect, perceived social support, sense of community, and life satisfaction. Comput Human Behav. 2014;30:69-78.

27. Akhavan-Malayeri F, NoghaniM, Khorasani MM. Social networks and happiness. Interdiscip Stud Media Cult. 2015;4(2):1-24.

28. Gentile B, Twenge JM, Freeman EC, Campbell WK. The effect of social networking websites on positive self-views: An experimental investigation. Comput Human Behav. 2012;28(5):1929-33.

29. Wood MA, Bukowski WM, Lis E. The Digital Self: How Social Media Serves as a Setting that Shapes Youth's Emotional Experiences. Adolesc Res Rev. 2016;1(2):163-73.

30. Wang JL, Jackson LA, Gaskin J, Wang HZ. The effects of Social Networking Site (SNS) use on college students' friendship and wellbeing. Comput Human Behav. 2014;37:229-36

31. Fathi S, Mokhtarpour M. Explanatory model of the Internet and its role in the cultural identity of students. J Cult Manag. 2013;7(19):11738.

32. Valkenburg PM, Peter J. Online communication among adolescents: An integrated model of its attraction, opportunities, and risks.J Adolesc Health. 2011;48(2):121-7.

33. TrepteS, Reinecke L. The reciprocal effects of social network site use and the disposition for self-disclosure: A longitudinal study. Comput Human Behav. 2013;29(3):1102-12.

34. Kearney CJ. Friendship quality, Facebook and self-concept: Social networking and adolescent development: Fairleigh Dickinson
University; 2012.

35. Lou LL, Yan Z, Nickerson A, McMorris R. An examination of the reciprocal relationship of loneliness and Facebook use among first-year college students. J Educ Comput Res . 2012;46(1):105-17.

36. Clayton RB, Osborne RE, Miller BK, Oberle CD. Loneliness, anxiousness, and substance use as predictors of Facebook use. Comput Human Behav. 2013;29(3):687-93.

37. Falsafi SG. Comparative approach to media literacy in developed countries. J Media Stud. 2014;9(24):151-66.

38. Salgado MV, Pérez-Stable EJ, Primack BA, Kaplan CP, Mejia RM, Gregorich SE, et al. Association of media literacy with cigarette smoking among youth in Jujuy, Argentina. Nicotine Tob Res. 2012;14(5):516-21.

39. Fingar KR, Jolls T. Evaluation of a school-based violence prevention media literacy curriculum. Inj Prev. 2014;20(3):183-90.

40. Khan S, Gagné M, Yang L, Shapka J. Exploring the relationship between adolescents 'self-concept and their offline and online social worlds. Comput Human Behav. 2016;55:940-5.

41. Hall RH. Internet Use and Happiness. Third International Conference on HCI in Business, Government and Organizations: eCommerce and Innovation. Toronto, Canada, 17-22 July 2016: 37-45.

42. Borca G, Bina M, Keller PS, Gilbert LR, Begotti T. Internet use and developmental tasks: Adolescents' point of view. Comput Human Behav. 2015;52:49-58.

43. Wu YJ, Outley C, Matarrita-Cascante D, Murphrey TP. A Systematic Review of Recent Research on Adolescent Social Connectedness and Mental Health with Internet Technology Use. Adolesc Res Rev. 2016;1(2):153-62.

44. Wierzańska M. Cyberspace as a current frame of reference for teenage life and values. Media I Społeczeństwo. 2012;2:147-56.

45. Farahmand M, Jvahrchyan N, Hatefirad L. Globalization of culture and rethinking sexual interaction. J Strateg Stu Glob. 2014;5(14):175204.

46. Kuss DJ, Van RooijAJ, Shorter GW, Griffiths MD, van de Mheen D. Internet addiction in adolescents: Prevalence and risk factors. Comput Human Behav. 2013;29(5):1987-96.

47. McCreanor T, Lyons A, Griffin C, Goodwin I, Moewaka Barnes H, Hutton F. Youth drinking cultures, social networking and alcohol marketing: Implications for public health. Crit Public Health. 2013;23(1):110-20.

48. Li C, Dang J, Zhang X, Zhang Q, Guo J. Internet addiction among Chinese adolescents: The effect of parental behavior and self-control. Comput Human Behav. 2014;41:1-7.

$49 \mathrm{Li} \mathrm{C}$, Tsai WHS. Social media usage and acculturation: A test with Hispanics in the US. Comput Human Behav. 2015;45:204-12.

50. Yao MZ, Zhong ZJ. Loneliness, social contacts and Internet addiction: A cross-lagged panel study. Comput Human Behav. 2014;30:164-70.

51. Mak KK, Lai CM, Watanabe H, Kim DI, Bahar N, Ramos M, et al. Epidemiology of internet behaviors and addiction among adolescents in six Asian countries. Cyberpsychol Behav Soc Netw. 2014;17(11):720-8.

52. Singleton A, Abeles P, Smith IC. Online social networking and psychological experiences: the perceptions of young people with mental health difficulties. Comput Human Behav. 2016;61:394-403. 